\title{
Eine Familie mit rezessiv-geschlechtsgebundener Taubstummheit ${ }^{*}$
}

\author{
Hisatoshi Mitsuda (Staatliche Medizinishe Korrektionsanstalt, Kyoto-Uji), \\ Syogo Inoue (Psychiatrische Klinik der Universität Mie) und \\ Yasuhiko Kazama (Ohren- und Hals-, Nasen-Klinik der Universität Mie)
}

Eingegangen am 25. Oktober 1952

UDC 575.115: 575.116. 7:

$575.181: 575.19$

Schon im Jahre 1921 äusserte Lenz die Vermutung, dass phänotypisch gleichen, bzw. ähnlichen Leiden nicht immer die gleiche genotypische Grundlage entspricht. Heute kennen wir bereits zahlreiche Erbkrankheiten, bei denen heterogene selbständige Formen beobachtet werden, welche sich klinisch etwas oder kaum unterscheiden lassen. Wir können demnach weiter vermuten, dass die Beispiele für die Heterogenie in Zukunft noch zunehmen werden, je gründlicher und eingehender man die einzelnen Leiden sowohl klinisch als auch genetisch studieren wird.

Für die erbliche Taubstummheit sind im allgemeinen zwei genetisch verschi dene Formen, d. h. die rezessive und die dominante (Typus Mondini), bekannt. Betreffs der rezessiven Taubstummheit haben jedoch Müh'mann (1930) sowie Grohmann (1939) auf Grund des Sippenbefundes das Vorliegen der Heterogenie zweier klinisch gleichartiger Krankheitsbilder nachgewiesen. Wir möchten nun ein $\mathbf{n}$ weiteren interessanten Stammbaum über Taubstummheit mitteilen, welche sicher einen ganz anderen Erbgang als bei den oben genannten Formen zeigt und sich als ein dritter Biotypus ausweisen dürfte.

\section{Sippenbefunde}

Eine ausgedehnte Sippenuntersuchung ergab folgende Einzelheiten, wobei die Ergebnisse der Untersuchung des Hörvermögens und der Labyrinthfunktion besonders in Tab. 1 zusammengefasst sind. Die in Klammer gesetzten Zahlen geben ihre Stellung in der Sippentafel (Abb. 1) an, die unnummerierten Sippenmitglieder sind angeblich alle hörgesund.

Der Proband (15), 20 Jahre alt; reif und glatt geboren, von Kindheit an taubstumm, scheint die Taubheit angeblich mit 4 Jahren aus Anlass eines heftigen Stosses gegen das rechte $\mathrm{Ohr}$ rasch verschlimmert $z$ u haben, obgleich er schon vorher kaum hören und sprechen konnte. Jedenfalls ist es nicht möglich, trotz dieser Angabe seiner Eltern, den Unfall als Ursache der Taubstummheit zu betrachten : Äusserer Gehörgang und Trommelfell zeigen beiderseits keinen krankhaften Befund. Neurologisch o. B., Intelligenz norma1.

Der Vater des Probanden (6), 64 Jahre alt, bekam vor 6 Jahren einen apoplektischen Anfall, leidet seitdem an linksseitiger Hemiplegie. Auch sein Hörvermögen hat seither abgenommen, wenngleich er vorher ganz hörgesund war.

\footnotetext{
* Herrn Prof. Dr. Lenz zum 65. Geburtstag überreicht von Dr. Med. H. Mitsuda.
} 


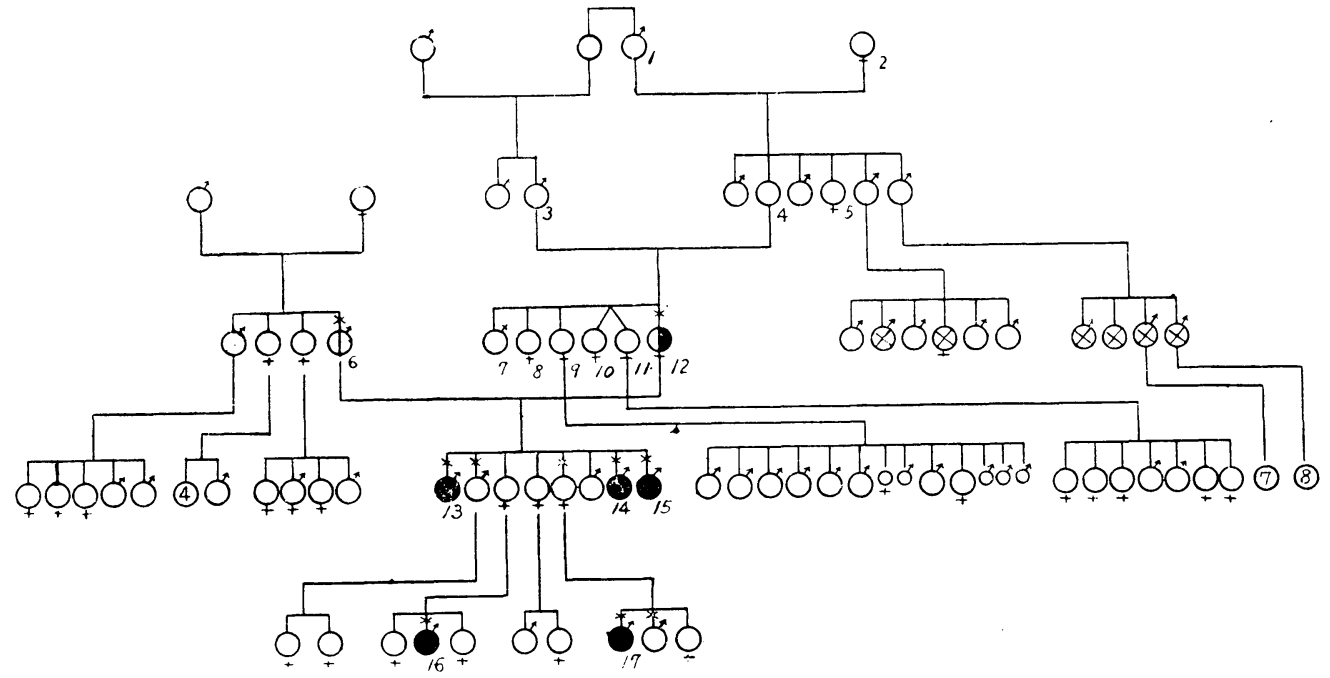

Abb. 1. Familie Om. mit rezessiv-geschlechtsgebundener Taubstummheit

- Taubstummheit

$\otimes$ Erblindung
- Taubheit (halbseitig)

주 우 klein gestorben
(1) Schwerhörigkeit

* selbst untersucht

Die Mutter des Probanden (12), 59 Jahre alt; ihrer eigenen Angaben nach soll die Taubheit, die sie vorher gar nicht bemerkt hatte, durch Sturz in einen Graben im Alter von 18 Jahren entstanden sein. Die Trommelfelle sind aber beiderseits in intaktem Zustand. Neurologisch o. B.

Der älteste Bruder des Probanden (13), 36 Jahre alt; angeboren taubstumm, hat keine nennenswerte Krankheit durchgemacht, ausser Krämpfen im Alter zwischen 3 und 4 Jahren. Ohrenärztlicher Befund ausser Hörvermögen und Labyrinthfunktion: Rechtes Ohr-kleine Perforation des Trommelfells mit schleimiger Sekretion. Diagnose : Akute eitrige Mittelohrentzündung (angeblich leidet er daran seit einer Woche). Linkes Ohr-narbig geheilter kleiner Defekt des Trommelfells. Neurologisch o. B., Intelligenz normal.

Der Bruder des Probanden (14), 21 Jahre alt; angeboren taubstumm, körperliche und psychische Entwicklung normal, hat lediglich Masern mit 3 Jahren überstanden. Sonstige Krankheiten wie Krämpfe, eitrige Ohrenleiden sollen nicht vorgekommen sein. Neurologisch o. B.

Der Neffe des Probanden (I6), 7 Jahre alt; angeboren taubstumm, reif und glatt geboren, Gehen erst nach dem Alter von 2 Jahren möglich, hat 2 Mal Pneumonie im Alter zwischen dem 9. Monat und 2 Jahren durchgemacht. Psychisch reizbar und explosiv, hat Neigung zu Pica, indem er gern Holzkohle, Asche usw. isst. Intelligenz mittelmässig, neurologisch o. B. Ohrenärztlicher Befund : beide Trommelfelle intakt; die Hörreste unklar, weil wir ihn diesbezüglich nicht untersuchen konnten: Beim Drehen nach rechts wie links wird kein Nachnystagmus konstatiert.

Der Neffe des Probanden ( 17 ), 8 Jahre alt; angeboren taubstumm, reif und glatt geboren, keine nennenswerte Krankheit durchgemacht. Die Trommelfelle sind beiderseits normal. Neurologisch und psychisch o. B. 


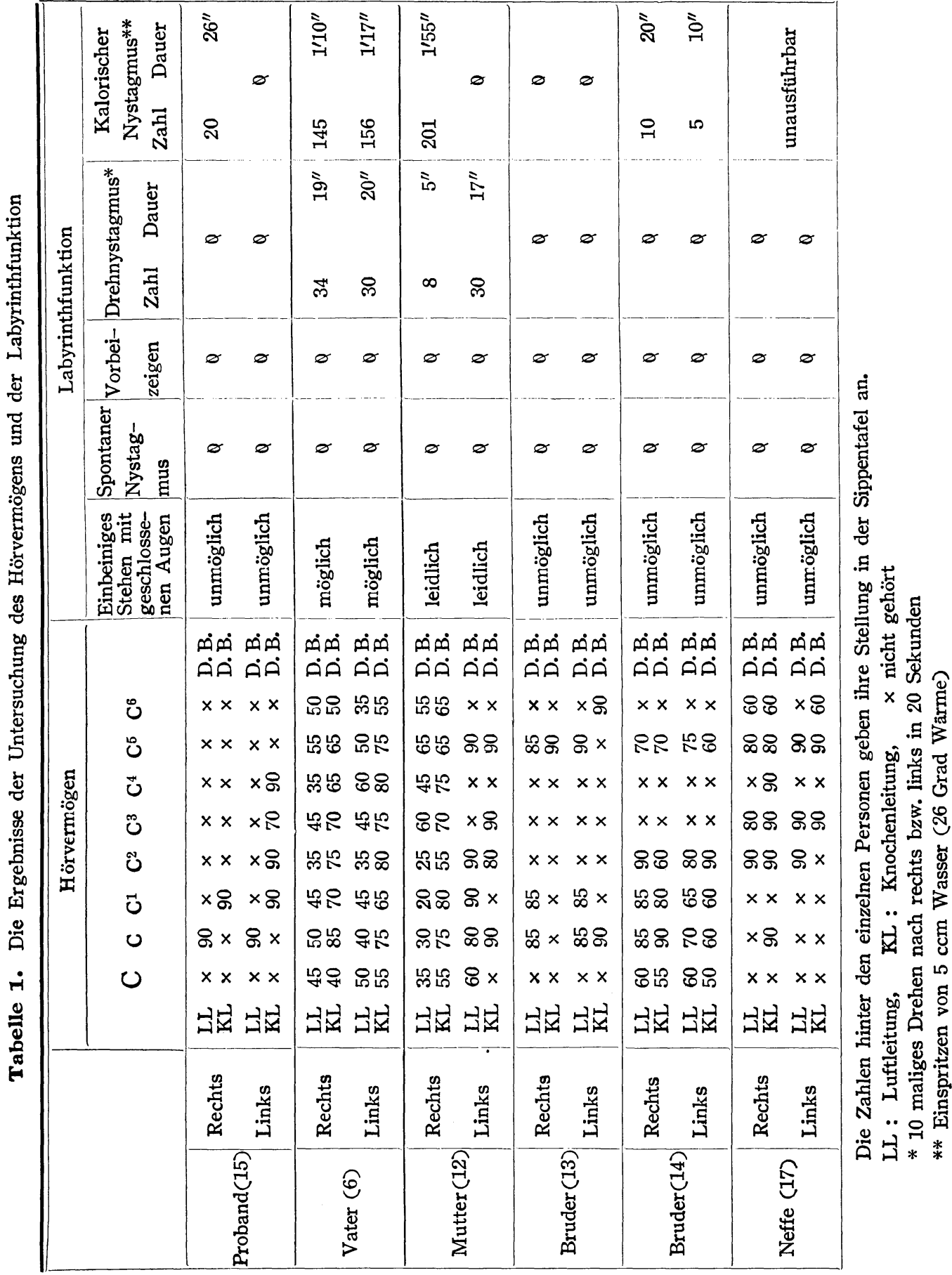

\section{Genotypische Betrachtung}

\section{Erbgang}

Ein Blick auf die Sippschaftstafel lässt erkennen, dass es sich hier um einen rezessivgeschlechtsgebundenen Erbgang handelt, da die Personen mit angeborener Taubstumm- 
heit alle männlich sind, indem von zwei Schwestern des Probanden, welche beide hörgesund sind, je ein taubstummer Knabe abstammt. Meines Wissens ist bis heute nur ein Stammbaum, nämlich der von G. S. Dow und C. I. Poynter berichtete "Dar Family" (1930), bekannt, in dem die Taubheit einen rezessiv-geschlechtsgebundenen Erbgang zeigte. In der betreffenden Familie waren 18 Taube, alle männlich, und drei eingeheiratete ebenfalls taube Frauen, die aber mit jenen männlichen Merkmalsträgern nicht blutsverwandt waren. Wir können aus dieser genealogischen Betrachtung von $z$ wei Stammbäumen den überzeugenden Beweis dafür liefern, dass für die erbliche Taubstummheit ausser einem einfachen rezessiven und dominanten Genotypus noch ein rezessiv-geschlechtsgebunder Typus vorhanden ist.

Es ist nun noch zu beachten, dass bei der Mutter des Probanden eine an Taubheit grenzende Schwerhörigkeit des linken Ohrs durch ohrenärztliche Untersuchung festgestellt worden ist, welche angeblich erst nach dem Sturz in einen Graben im Alter von 18 Jahren entstanden sein soll. H. Grohmann hat die Vermutung geäussert, dass heterozygote Anlageträger für rezessive Taubstummheit nach einer exogenen Schädigung ihres Gehörorgans eher als Erbgesund ertauben. So dürfte es nicht ganz unberechtigt sein anzunehmen, dass es sich bei dieser Mutter um die phänotypische Manifestierung der latenten Anlage zur Taubheit durch ein exogenes Moment handelt, weil die Mutter des Probanden gemäss dem rezessiv-geschlechtsgebundenen Erbgang sicher als Konduktorin anzusehen ist.

\section{Ursprung der Mutation}

Es ist bekannt, dass man im günstigen Fall, meistens bei geschlechtsgebunden-rezessiver Vererbung, den Ursprung der Genmutation eines Erbleidens unter den Mitgliedern einer Sippschaft ungefähr feststellen kann. So haben z. B. Boggs sowie Rewcastle eine solche Ansicht auf Grund des Sippenbefundes von Hämophilie geäussert. Ü̈berblicken wir nun die vorliegende Familie im ganzen, so sehen wir in der Descendenz einer nicht blutsverwandten Ehe ( 6 und 12) plötzlich drei taubstumme Knaben auftreten, wobei wir unter den Vorfahren keine Kranken finden. Hier müssen wir noch in Betracht ziehen, dass wir die Mutter des Probanden, wie schon erwähnt wurde, als Anlageträgerin anzusehen haben. Auf Grund dieser Indizien scheint es uns möglich, entweder bei der Grossmutter des Probanden (4) oder dem Urgrossvater des Probanden (1) den fraglichen Ursprung der Mutation des Gens für die rezessiv-geschlechtsgebundene Taubstummheit anzunehmen. Welche von beiden Möglichkeiten wahrscheinlicher ist, können wir nicht mit Bestimmtheit sagen, da die Grossmutter des Probanden (4) nur eine Schwester (5) besitzt, die leider kein Kind hat. Den Grossvater (3) können wir als sehr unwahrscheinlich ausschalten, weil die zahlreichen Söhne seiner zwei Töchter (9) (11) alle hörgesund sind. Anderseits wäre es allerdings nicht unmöglich, dass die Urgrossmutter (2) selbst als Konduktorin die betreffende Anlage schon ron ihren Vorfahren her vererbt hätte. Es scheint aber die Tatsache, dass wir unter ihren vier Söhnen keinen einzigen Merkmalsträger finden, nicht für sondern eher gegen diese Annahme zu sprechen. 
Hinzugefügt sei noch, dass das Dorf, wo die Familie des Probanden ansässig ist, eine Einwohnerzahl von 3721 hat, und dass darunter, abgesehen von den hier aufgeführten Kranken, noch an drei Personen Taubheit festgestellt wird, welche aber von sporadischer Natur ist.

\section{Phänotypische Betrachtung}

Schon seit der Untersuchung von Hugo Frey und Hammerschlag ist bekannt, dass sog. "Drehversager" bei angeborener Taubheit viel seltner sind als bei in früher Kindheit erworbener. Weiter nimmt man an, dass auch diejenige Fälle von angeborener Taubheit, die Störungen des Gleichgewichtsapparates aufweisen könnten, auf intrauterine Erkrankungen oder Geburtstraumen zurückzuführen sein dürften.

E. Müller (1937) hat aber in zwei Familien hereditäre Taube, bzw. Schwerhörige mit Schädigung des Vorhofbogengangsapparates beobachtet und will zeigen, dass aus der Art der Vestibularissörung keinesfalls Rückschlüsse auf die Ätiologie des Leidens gezogen werden können, ja dass selbst Lagenystagmus die Erblichkeit einer Taubheit nicht ausschliesst. Wir dürfen aber nicht übersehen, dass die zwei Familien Müllers genealogisch sicher andere Sippenbefunde als die der üblichen rezessiven Formen zeigen, indem eine direkte Übertragung des Leidens von den Eltern auf das Kind in beiden Familien vorliegt. Müller selbst hat die Taubheit in der einen Familie als dominanten hereditär-degenerativen Typus angesehen, während er in der anderen Familie als Ursache der Taubheit bei dem Vater eine exogene Schädigung angenommen hat, um damit hier das Vorliegen des rezessiven Typus nachzuweisen.

Wie dem auch sei, scheint jedenfalls erwiesen zu sein, dass in typischen Fällen von rezessiver Taubstummheit der Vestibularapparat im allgemeinen durchaus funktionstiichtig ist. Bemerkenswert ist in diesem Zusammenhang, dass wir bei allen taubstummen Personen in_der vorliegenden Familie mehr oder weniger Schädigung des Vorhofbogengangsappartes feststellen konnten. Die Prüfungen der Labyrinthfunktion ergaben bei allen taubstummen Personen keine bzw. eine deutlich herabgesetzte Erregbarkeit, indem Drehnystagmus bei keiner Person und kalorischer Nystagmus bei zwei von drei Geschwistern einschliesslich des Probanden nur spurweise, bei einem anderen Fall gar nicht konstatiert wurden. Ausserdem war einbeiniges Stehen mit geschlossenen Augen bei allen Kranken unmöglich, wenngleich wir bei ihnen weder spontanen Nystagmus noch Vorbeizeigen feststellen konnten. Auf Grund dieser Beobachtungen können wir schliessen, dass sich die rezessiv-geschlechtsgebundene Taubstummheit als ein besonderer Biotypus von der einfachen rezessiven auch in ihrer phänotypischen Manifestation unterscheiden dürfte.

Weiterhin hat Schwarz darauf hingewiesen, dass, in Fällen von Taubstummheit in Verbindung mit Retinitis pigmentosa gelegentlich stärkere Veränderungen in Labyrinth beobachtet werden. Wenngleich wir in dieser Arbeit den Augenhintergrund nicht untersuchen konnten, scheint doch die Tatsache, dass bei allen taubstummen Personen, besonders bei dem 36 jährigen Bruder des Probanden, noch keine Sehstörungen bemerkt wurden, eher gegen das Vorhandensein von Retinitis pigmentosa zu sprechen. 


\section{Zusammenfassung}

1. $\mathrm{Zu}$ dem bisher von G. S. Dow und C. I. Poynter berichteten einzigen Fall von rezessiv-geschlechtsgebundener Taubstummheit wird hier ein weiteres Beispiel beschrieben.

2. Wir vermuten, dass sich dieser Biotypus auch in seiner phänotypischen Manifestierung von der einfachen rezessiven unterscheidet, indem sich bei allen taubstummen Personen der vorliegenden Familie deutlich Störungen der Labyrinthfunktion festsellen lassen.

3. Es wurde der Versuch gemacht, unter den Mitgliedern der Sippe den möglichen Ursprung der Mutation des betr. Gens festzustellen.

\section{Literatur}

Boggs, R.: Zit. nach R. Gates. Dow, G. S. u. C. I. Poynter: The Eugenical News 15 (1930). Gates, R.: Human Genetics (1946). Grohmann, H. : Der Erbarzt 7 (1939). Lenz, F.: Z. Abstamm. lehre 27 (1921). Mühlmann, W. E.: Arch. Rassenbiol. 22 (1930). Müller, E.: Arch. f. Ohren-. u. Kehlkopfkunde 142 (1937). Rewcastle, A. G.: Zit. nach R. Gates. Schwarz, M.: Deutsche Med. Wschr. 62 (1936).

\section{伴性劣性の霆丝の一家系}

本家系の管咧は伴性劣性の遗伝様式を示す点に興味がある. この種の症例は Dow 之 Poynter

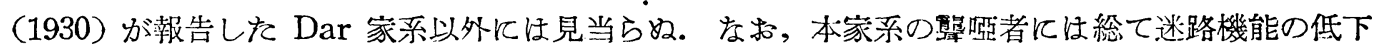

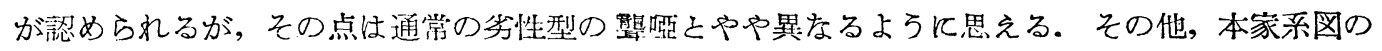

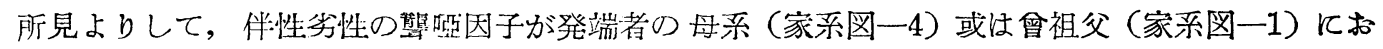
んて突然変異の結果発現したのではないかと推測される。但し，會祖母（家系図一2）に該因子が 既に潜在していたとも考えられるが，そのような可能性任彼女の四人の男児が総て正常である点 よりして, 非常に尠んもの亡言えよう.な撜端者の母の聚 (片側) は外傷により後天的に獲得 されたものではあるが，Grohmann 等の見解よりしても，潜在性因子による素地の意味をその祭 一応教虑するととも必要であろう。 